\title{
HIGH-RESOLUTION PHOTOACOUSTIC TOMOGRAPHY
}

\author{
Lihong V. Wang*, Xueding Wang, Geng Ku, Xueyi Xie, George Stoica \\ Optical Imaging Laboratory, Department of Biomedical Engineering \\ Texas A\&M University, 3120 TAMU, College Station, Texas 77843-3120 \\ URL: http://oilab.tamu.edu; Email: LWang@tamu.edu \\ * Corresponding author.
}

\begin{abstract}
Optical contrast is sensitive to physiological parameters, such as the oxygen saturation and total concentration of hemoglobin, in biological tissues. Photoacoustic tomography is based on the high optical contrast yet utilizing the high ultrasonic resolution. Our work in this emerging area of research will be summarized in this invited talk. In this technology, a diffraction-based inverse-source problem is solved in the image reconstruction, for which we developed the rigorous reconstruction theory. We implemented a prototype and accomplished non-invasive transdermal and transcranial functional imaging of small-animal brains in vivo. Changes in the cerebral blood oxygenation and blood volume of a rat, as a result of the alternation from hyperoxia to hypoxia, were imaged successfully.
\end{abstract}

Keywords - Photoacoustic tomography; optical contrast; ultrasonic resolution; inverse-source reconstruction; oxygen saturation of hemoglobin

\section{INTRODUCTION}

Photoacoustic tomography (PAT, also referred to as optoacoustic or thermoacoustic tomography) involves both photons and ultrasound. A short-pulsed laser source is used to irradiate the biological tissue samples under investigation. A temperature rise of the order of $\mathrm{mK}$ will be produced in a short time frame. Consequently, thermoelastic expansion will cause emission of acoustic waves, referred to as photoacoustic waves. The photoacoustic waves are measured by wideband ultrasonic transducers around the sample, and the acquired photoacoustic waves are used to reconstruct the optical absorption distributions. Because the laser pulse is short, proportionately high frequency ultrasonic waves will be produced, which can provide diffraction-limited spatial resolution. In summary, the contrast in PAT is determined primarily by the optical properties of the biological tissues, and the spatial resolution in PAT is determined primarily by the photoacoustic waves originating from within the biological tissues.

\section{FUNDAMENTALS OF PHOTOACOUSTICS: RECONSTRUCTION ALGORITHM}

If the electromagnetic pumping pulse duration is much shorter than the thermal diffusion time through a voxel, thermal diffusion can be neglected; this is known as the thermal confinement. In this case, the acoustic wave $p(\mathbf{r}, \bar{t})$ is related to electromagnetic absorption, $H(\mathbf{r}, \bar{t})$, by the following wave equation:

$$
\frac{\partial^{2} p(\mathbf{r}, \bar{t})}{\partial \bar{t}^{2}}-\nabla^{2} p(\mathbf{r}, \bar{t})=\frac{\beta v_{s}}{C} \frac{\partial H(\mathbf{r}, \bar{t})}{\partial \bar{t}},
$$

where $\bar{t}=t v_{s} ; \quad v_{s}$ is the acoustic speed, assumed to be constant; $C$ is the specific heat; and $\beta$ is the coefficient of volume thermal expansion. $H(\mathbf{r}, \bar{t})$ can further be written as the product of a purely spatial component $\varphi(\mathbf{r})$ that describes the electromagnetic absorption properties of the medium at $\mathbf{r}$ and a purely temporal component $\eta(\bar{t})$ that describes the shape of the irradiating pulse.

Our group derived the exact inverse solutions in planar, spherical, and cylindrical geometries [1-3]. When the distance between the photoacoustic sources and the detector is much longer than the wavelengths of the highfrequency photoacoustic waves that are useful for imaging, the following approximate inverse solution holds:

$$
\varphi(\mathbf{r})=\left.C \iint_{S_{0}} d S_{0} \cos \left(\theta_{d}\right) \frac{1}{t} \frac{\partial p\left(\mathbf{r}_{0}, t\right)}{\partial t}\right|_{t=\mid \mathbf{r}_{0}-\mathbf{r} / / v_{s}},
$$

where $C$ is a constant; $S_{0}$ is the surface of detection; and $\theta_{d}$ is the angle between the normal of $d S_{0}$ and $\mathbf{r}-\mathbf{r}_{0}$.

\section{EXPERIMENTS}

The experimental system for PAT of a rat brain in vivo has been described before [see Ref. (4)]. In this work, through the modulation of the inhaled oxygen concentration, we changed the systemic physiological status of the rat. Firstly, provided with pure oxygen, the rat was under the hyperoxia status. Two photoacoustic images of optical absorption in the cerebral cortex of the rat brain were acquired with laser light at the $584-\mathrm{nm}$ and the $600-\mathrm{nm}$ wavelengths, respectively [see Figs. 1(A) and (B)]. Then the breathing gas was changed slowly to a mixed gas with a low concentration of oxygen $\left(\sim 8 \% \mathrm{O}_{2}\right.$, $\sim 5 \% \mathrm{CO}_{2}$ and $\sim 87 \% \mathrm{~N}_{2}$ ). Then, under the hypoxia status, another two images of the cerebral cortex corresponding to the same two wavelengths were acquired (not shown). With the high optical contrast between the blood and background brain tissues, each brain image presents the vascular structure in the cerebral cortex clearly and matches well with the open-skull anatomical photograph obtained after the photoacoustic imaging experiment [see 
Fig. 1(G)].

For each physiological status, the two images of the relative optical absorptions at the two wavelengths enable us to compute the point-by-point hemoglobin oxygen saturation $\left(\mathrm{SO}_{2}\right)$ and total concentration of hemoglobin $(\mathrm{HbT})$ in the cortical venous vessels through the following equations:

$$
\begin{gathered}
\mathrm{SO}_{2}=\frac{\left[\mathrm{HbO}_{2}\right]}{\left[\mathrm{HbO}_{2}\right]+[\mathrm{Hb}]}=\frac{\mu_{a}^{\lambda_{2}} \varepsilon_{\mathrm{Hb}}^{\lambda_{1}}-\mu_{a}^{\lambda_{1}} \varepsilon_{\mathrm{Hb}}^{\lambda_{2}}}{\mu_{a}^{\lambda_{1}} \varepsilon_{\Delta \mathrm{Hb}}^{\lambda_{2}}-\mu_{a}^{\lambda_{2}} \varepsilon_{\Delta \mathrm{Hb}}^{\lambda_{1}}}, \\
\mathrm{HbT}=\left[\mathrm{HbO}_{2}\right]+[\mathrm{Hb}]=\frac{\mu_{a}^{\lambda_{1}} \varepsilon_{\Delta \mathrm{Hb}}^{\lambda_{2}}-\mu_{a}^{\lambda_{2}} \varepsilon_{\Delta \mathrm{Hb}}^{\lambda_{1}}}{\varepsilon_{\mathrm{Hb}}^{\lambda_{1}} \varepsilon_{\mathrm{HbO}}-\varepsilon_{\mathrm{Hb}}^{\lambda_{2}} \varepsilon_{\mathrm{HbO}}^{\lambda_{1}}},
\end{gathered}
$$

where $\mu_{a}$ is the absorption coefficient; $\lambda_{1}$ and $\lambda_{2}$ are the two wavelengths; $\varepsilon_{\mathrm{Hb}}$ and $\varepsilon_{\mathrm{HbO} 2}$ are the known molar extinction coefficients of the oxy- and deoxy-hemoglobin, respectively; and $[\mathrm{Hb}]$ and $\left[\mathrm{HbO}_{2}\right]$ are, respectively, the concentrations of the two forms of hemoglobin.

The functional images of $\mathrm{SO}_{2}$ and $\mathrm{HbT}$ corresponding to the two statuses are shown in Figs. 1(C)(F). When the status of the rat was altered from the hyperoxia to the hypoxia, the $\mathrm{SO}_{2}$ in the cerebral cortical venous vessels decreased. The average $\mathrm{SO}_{2}$ levels were $\sim 86 \%$ and $\sim 61 \%$ under the hyperoxia status and the hypoxia status, respectively. Simultaneously, the $\mathrm{HbT}$ in the cerebral cortical venous vessels increased as a result of this alternation. The average $\mathrm{HbT}$ value under the hypoxia status was about $\sim 11 \%$ greater than that under the hyperoxia status.
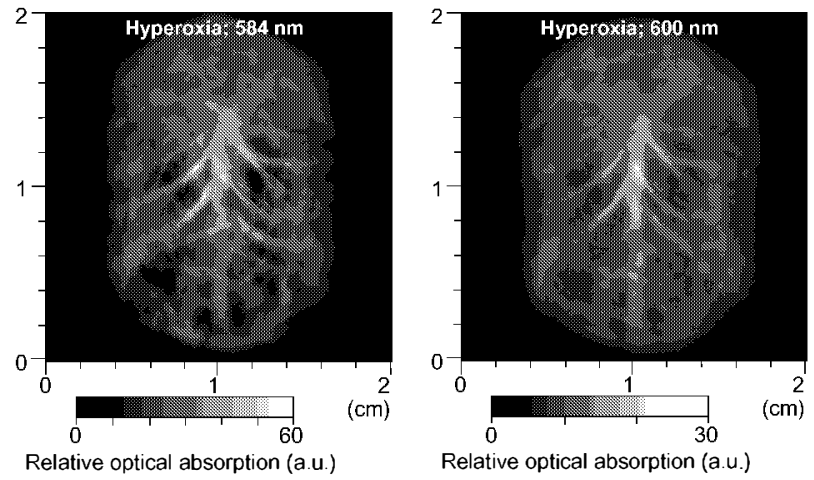

(A)

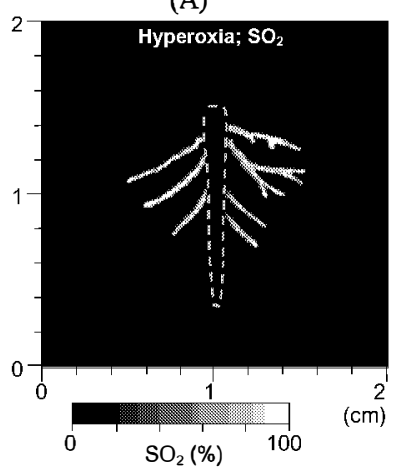

(C)

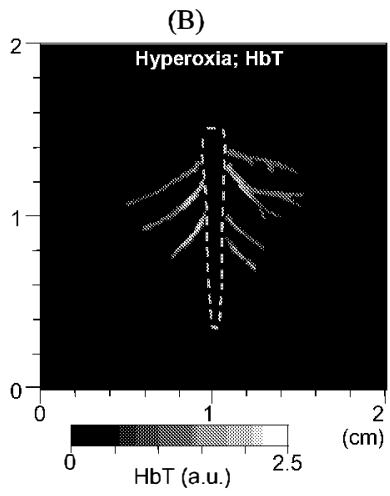

(D)

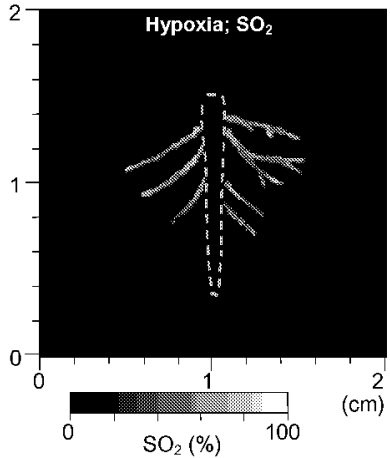

(E)

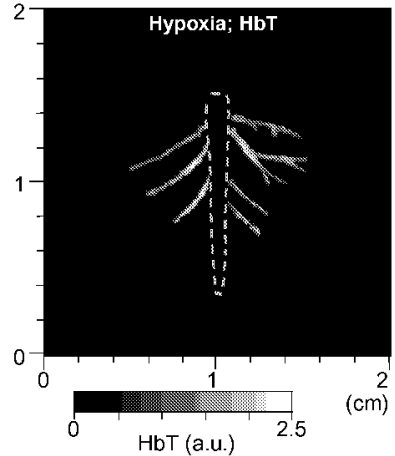

(F)

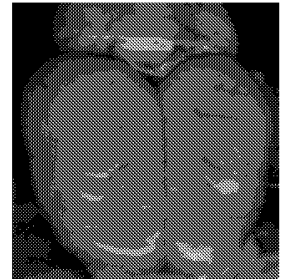

(G)

Fig. 1 (A) and (B), Images of the cerebral cortex of a rat brain generated by the $584-\mathrm{nm}$ and $600-\mathrm{nm}$ laser light respectively under the hyperoxia status. (C) and (D), Images of $\mathrm{SO}_{2}$ and $\mathrm{HbT}$ in the cortical venous vessels under the hyperoxia status. (E) and (F), Images of $\mathrm{SO}_{2}$ and $\mathrm{HbT}$ under the hypoxia status. (G), Open-skull anatomical photograph of the rat brain.

\section{SUMMARY}

We developed rigorous reconstruction algorithms, the foundation for PAT. We accomplished non-invasive transdermal and transcranial imaging of small-animal brains in vivo showing both structures and functions. Based on the spectroscopic differences between oxy- and deoxy-hemoglobins, PAT can enable accurate functional imaging of cerebral blood oxygenation and blood volume in small-animal brains with satisfactory spatial resolution.

\section{ACKNOWLEDGEMENTS}

This project was sponsored in part by the U.S. Army Medical Research and Materiel Command Grant No. DAMD17-00-1-0455, the National Institutes of Health Grants No. R01 EB000712 and No. R01 NS46214, and Texas Higher Education Coordinating Board Grant No. ARP 000512-0063-2001.

\section{REFERENCES}

1. M. Xu and L.-H. Wang, "Time-domain reconstruction for thermoacoustic tomography in a spherical geometry," IEEE T. Med. Imag. 21, 814-822 (2002).

2. Y. Xu, D. Feng, and L.-H. Wang, "Exact frequency-domain reconstruction for thermoacoustic tomography-I: Planar geometry," IEEE T. Med. Imag. 21, 823-828 (2002).

3. Y. Xu, M. Xu, and L.-H. Wang, "Exact frequency-domain reconstruction for thermoacoustic tomography-II: Cylindrical geometry," IEEE T. Med. Imag. 21, 829-833 (2002).

4. X. Wang, Y. Pang, G. Ku, X. Xie, G. Stoica, and L.-H. Wang, "Noninvasive laser-induced photoacoustic tomography for structural and functional imaging of the brain in vivo," Nat. Biotech. 21, 803-806 (2003). 